\title{
Role of Intra-abdominal Pressure in Early Acute Kidney Injury: A Prospective Cohort Study in Critically III Obstetric Patients
}

\author{
Asha Tyagi, Shubham Lahan, Gaurav Verma, Shukla Das, Mahendra Kumar \\ Department of Anaesthesiology and Critical Care, University College of Medical Sciences and GTB Hospital, New Delhi, India
}

Abstract

\begin{abstract}
Aims: This prospective cohort study evaluated intra-abdominal pressure (IAP) and its role in causing acute kidney injury (AKI) in critically ill obstetric patients and utility of urinary neutrophil gelatinase-associated lipocalin (NGAL) to predict AKI. Methods: A total of 50 eligible obstetric patients admitted to our Intensive Care Unit were enrolled and daily IAP measured using indwelling Foley catheter. Early AKI was diagnosed as per the KDIGO criteria and urine assessed for NGAL using ELISA. Results: AKI was seen in 54\% and intra-abdominal hypertension (IAH) in $21 \%$ patients. In patients with and without AKI, there was statistically similar IAP on day $1(P=0.542)$ and day $2(P=0.907)$ as well as incidence of IAH $(19 \%$ vs. $23 \%)(P=0.766)$. Area under receiver operating characteristic curve (AUC) for IAP to predict early AKI was $0.499(95 \%$ confidence interval $[\mathrm{CI}]: 0.325-0.673)(P=0.992)$. Urinary NGAL concentration was significantly greater in patients with early AKI compared to those without $(P=0.006)$; AUC for urinary NGAL to detect early AKI was $0.734(95 \% \mathrm{CI}: 0.583-0.884)(P=0.006)$ and optimal cutoff was $53.7 \mathrm{ng} / \mathrm{ml}$. Conclusions: IAH and AKI are common in critically ill obstetric patients. While IAP does not correlate with
\end{abstract} early AKI, NGAL is useful to predict AKI.

Keywords: Acute kidney injury, intra-abdominal hypertension, intra-abdominal pressure

\section{INTRODUCTION}

Intra-abdominal pressure (IAP) is defined as the steady state pressure contained within the abdominal cavity. ${ }^{[1]}$ It is best measured as the intravesical pressure through an indwelling Foley bladder catheter. ${ }^{[1]}$ Normal value of IAP in apparently healthy individuals varies between 0 and $5 \mathrm{mmHg},{ }^{[2]}$ and in critically ill patients admitted to Intensive Care Unit (ICU) it is slightly higher, namely, 5-7 mmHg. ${ }^{[1]}$ Values of IAP $\geq 12 \mathrm{mmHg}$ indicate intra-abdominal hypertension (IAH), and an increase to $>20 \mathrm{mmHg}$ along with a new-onset organ dysfunction depicts abdominal compartment syndrome, the most severe form of IAH. ${ }^{[1]}$ IAH is seen in up to $80 \%$ of critically ill patients ${ }^{[3]}$ and is associated with higher incidence of multiorgan failure and mortality as well as longer duration of stay in the ICU. ${ }^{[3]}$

The adverse effect of IAH on renal function, in particular, is well established. ${ }^{[4]}$ It is documented that not only IAH but even moderate rise in IAP of $8 \mathrm{mmHg}$ or greater can be associated with deterioration in renal functions. ${ }^{[4]}$ To diagnose an acute deterioration in renal function serum creatinine and/or urine output are conventionally relied on and be nonspecific as well.

\begin{tabular}{|l|l|}
\hline \multicolumn{3}{c|}{ Access this article online } \\
\hline Quick Response Code: & Website: \\
& www.ijccm.org \\
& \\
\end{tabular}

However, both these parameters are known to change late in the course of renal insult. Neutrophil gelatinase-associated lipocalin (NGAL) is a newer urinary biomarker to detect acute kidney injury (AKI) that shows an early increase in levels within $6 \mathrm{~h}$ of renal injury. ${ }^{[5]}$

Obstetric patients constitute a special group of critically ill patients in the ICU with regard to IAP. There are some previous studies noting raised IAP in term parturients as well as in those obstetric patients admitted to ICU after delivery. ${ }^{[6]}$ Even though raised IAP is documented in obstetric patients, it remains to be established whether this is merely a physiologic alteration due to the gravid uterus or is associated with adverse effects on organ functions. ${ }^{[7]}$

In particular, there are no studies in critically ill obstetric patients evaluating the consequences of IAP on renal function.

Address for correspondence: Dr. Asha Tyagi,

103, Siddhartha Enclave, Near Ashram Chowk, New Delhi - 110 014, India. E-mail: drashatyagi@gmail.com

This is an open access journal, and articles are distributed under the terms of the Creative Commons Attribution-NonCommercial-ShareAlike 4.0 License, which allows others to remix, tweak, and build upon the work non-commercially, as long as appropriate credit is given and the new creations are licensed under the identical terms.

For reprints contact: reprints@medknow.com

How to cite this article: Tyagi A, Lahan S, Verma G, Das S, Kumar M. Role of intra-abdominal pressure in early acute kidney injury: A prospective cohort study in critically Ill obstetric patients. Indian J Crit Care Med 2018;22:602-7. 
Against this background, we evaluated IAP and its effect on renal function in critically ill obstetric patients. The utility of urinary NGAL to predict acute deterioration in renal function in these settings was also assessed. Evaluation of renal function herein included early AKI as defined by the KDIGO guidelines. ${ }^{[8]}$ In addition, given the paucity of data, epidemiology of IAH was also assessed for the duration of ICU stay.

\section{Methods}

This prospective, noninterventional, observational cohort study was undertaken after approval from the Institutional Ethics Committee-Human Research in its meeting held on June 1, 2016 and obtaining informed written consent from caregiver of all the included patients. It was conducted from June 2016 to March 2017.

All obstetric patients admitted to our multidisciplinary ICU during pregnancy or up to 6 weeks after delivery of baby, for causes related directly or indirectly to pregnancy, were included in the study. The usual indications for ICU admission in obstetric patients are preeclampsia, obstetric hemorrhage, or sepsis. Those with anticipated duration of stay of $<24 \mathrm{~h}$, or not requiring a Foley bladder catheter, or with any contraindication to IAP measurements, that is, pelvic fracture or bladder injury, or with evidence of chronic renal disease were excluded.

\section{Method for measuring intra-abdominal pressure}

IAP was measured through a Foley catheter using previously recommended method of intravesical pressure monitoring using a pressure transducer. ${ }^{[1]}$ Transducer was zeroed at the level of midaxillary line at the iliac crest. The IAP was recorded during end expiration after injecting $25 \mathrm{ml}$ of saline into the bladder with patient in supine position. For checking that the pressure signal was correctly transduced, gentle compressions of the abdomen were seen to cause instant oscillation in the IAP tracing. If the signal appeared damped, the Foley catheter was opened to flush out airway bubbles and the procedure repeated. The absence of any active abdominal contractions/straining was ensured during measurement, including the use of sedation if required. All measurements were made by one of two investigators.

The IAP was measured twice daily, once each in morning and evening and averaged to represent the "daily IAP." The daily IAP was recorded for entire duration or first 7 days of ICU stay, whichever was earlier. The severity of IAH was quantified as Grade I: $12-15 \mathrm{mmHg}$; Grade II: 16-20 mmHg; Grade III: 21-25 mmHg; or Grade IV: > $25 \mathrm{mmHg} .^{[1]}$

\section{Diagnosis of acute kidney injury}

Renal dysfunction was diagnosed as the presence of AKI within the first 2 days of ICU stay (i.e., early). Hourly urine output and daily serum creatinine were used for diagnosing AKI as per the KDIGO Clinical Practice Guidelines ${ }^{[8]}$ that is, an increase in serum creatinine $\geq 0.3 \mathrm{mg} / \mathrm{dl}$ within $48 \mathrm{~h}$, or an increase in serum creatinine to $\geq 1.5$ times baseline which is known or presumed to have occurred within the prior 7 days, or urine volume $<0.5 \mathrm{ml} / \mathrm{kg} / \mathrm{h}$ for $6 \mathrm{~h}$. Baseline serum creatinine was recorded from patients' preoperative records, and if not available, was estimated using the modification of diet in renal disease (MDRD) equation ${ }^{[9]}$ to enable detection of AKI. The MDRD equation assumed a lower limit of normal baseline glomerular filtration rate of $75 \mathrm{ml} /$ minute per $1.73 \mathrm{~m}^{2}$.

The severity of AKI was graded as per the guidelines, namely, Stage 1: serum creatinine of 1.5-1.9 times, the baseline value or $>0.3 \mathrm{mg} / \mathrm{dl}$ rise, or urine output $<0.5 \mathrm{ml} / \mathrm{kg} / \mathrm{h} \mathrm{h}$ for 6-12 h; Stage 2: 2.0-2.9 times rise in serum creatinine or urine output $<0.5 \mathrm{ml} / \mathrm{kg} / \mathrm{h} \mathrm{h}$ for $\geq 12 \mathrm{~h}$; and Stage 3: serum creatinine increasing 3 times the baseline or $\geq 4.0 \mathrm{mg} / \mathrm{dl}$ or urine output $<0.3 \mathrm{ml} / \mathrm{kg} / \mathrm{h} \mathrm{h}$ for $\geq 24 \mathrm{~h}$ or anuria $\geq 24 \mathrm{~h}{ }^{\left[{ }^{8]}\right.}$

\section{Method for urinary neutrophil gelatinase-associated lipocalin estimation}

Urine sample was collected on ICU admission for estimation of urinary NGAL. The urine samples were centrifuged and frozen at $-80^{\circ} \mathrm{C}$ till assayed. A commercial enzyme-linked immunosorbent assay (ELISA) kit (NGAL ELISA, Biovendor, Czech Republic) was used for the estimation of urinary NGAL as per the manufacturer's details. This kit had a minimum detection level of $0.02 \mathrm{ng} / \mathrm{ml}$.

\section{Ancillary observations}

For evaluating epidemiology of IAH in critically ill obstetric patients, its associated risk factors ${ }^{[1]}$ were noted for the entire duration of ICU stay or first 7 days whichever was earlier. These included the following major risk factors: (a) abdominal surgery (cesarean section or laparotomy), (b) major trauma, (c) ileus defined as abdominal distension or absence of bowel sound or failure enteral feeding, (d) clinically diagnosed abdominal distension, (e) intraperitoneal infection or collection, (f) liver dysfunction or cirrhosis with ascites, (g) massive fluid resuscitation defined as intravenous (IV) infusion of $>3.51$ of IV fluid per day, and (h) polytransfusion defined as $>6$ units of blood or blood products per day as well as minor risk factors: (a) sepsis, (b) mechanical ventilation (noninvasive or invasive), (c) positive end-expiratory pressure $>10 \mathrm{cmH}_{2} \mathrm{O}$, (d) body mass index (BMI) $>30 \mathrm{~kg} / \mathrm{m}^{2}$, (e) coagulopathy, (f) acidosis $(\mathrm{pH}<7.2)$, and $(\mathrm{g})$ hypothermia $\left(<33^{\circ} \mathrm{C}\right)$.

Relevant patient characteristics and obstetric details including age, BMI, period of gestation (weeks), reason for ICU admission, mode of fetal delivery as well as sequential organ failure assessment (SOFA) score on day of ICU admission, ICU mortality, and duration of stay in ICU were also recorded.

\section{Statistical analysis}

To evaluate the effect of IAP on renal dysfunction, IAP was compared for patients with and without AKI and a receiver operating characteristic (ROC) curve was constructed between the IAP and presence of early AKI. Since we evaluated only early AKI (within the first 2 days of ICU admission), both 
analyses included IAP on day 1 and day 2 of ICU stay only. Utility of urinary NGAL to predict early AKI was analyzed by comparing values of the biomarker between patients with and without AKI and also constructing a ROC curve for the diagnostic performance of urinary NGAL for predicting early AKI. Data are represented as mean \pm standard deviation or median (interquartile range) for normal and nonnormal distribution respectively. Comparison of quantitative data was done using $t$-test or Mann-Whitney U-test for parametric and nonparametric data, respectively, and the Chi-square or Fischer's exact test for qualitative data. Normality of data was assessed using the Kolmogorov-Smirnov test. The data were analyzed using IBM SPSS (version 23.0) and $P<0.05$ was taken as statistically significant.

Sample size: This cohort aimed to include a minimum of 50 patients, taking into consideration the planned duration of the study.

\section{RESULTS}

During the study period, a total of 82 obstetric patients were admitted to the ICU of which 66 were assessed for eligibility. From among the 66 patients, 16 were excluded due to refusal of consent $(n=2)$, duration of ICU stay $<24 \mathrm{~h}(n=9)$, or fatal outcome within $<24 \mathrm{~h}(n=5)$. We included the remaining 50 patients, but the urine samples of 2 were misplaced, and hence the analysis is presented for 48 patients.

The patient characteristics and other details of the cohort are depicted in Table 1 .

Of the $40 / 48$ patients who had a surgical intervention [Table 1], majority $(37 / 40=92 \%)$ underwent a cesarean section, while remaining $(3 / 40=8 \%)$ had an emergency laparotomy following postpartum hemorrhage.

The reason for ICU admission included preeclampsia $(33 / 48=69 \%)$, antepartum or postpartum hemorrhage $(7 / 48=15 \%)$, severe anemia unrelated to obstetric blood loss $(4 / 48=8 \%)$, sepsis $(3 / 48=6 \%)$, and hepatic encephalopathy $(1 / 48=2 \%)$.

Table 1: Characteristics of obstetric patients admitted to the Intensive Care Unit $(n=48)$

\begin{tabular}{lc}
\hline Characteristic & Value \\
\hline Age (years) & $25 \pm 4.3$ \\
BMI; kg/m² & $22.2 \pm 3$ \\
Period of gestation; weeks & $34.7 \pm 3.8$ \\
Pregnant at time of ICU admission & $1 / 48(2)$ \\
Surgical intervention & $40 / 48(83)$ \\
SOFA score & $6(4.2-8.7)$ \\
Duration of ICU stay; days & $2.5(2-4)$ \\
ICU-mortality & $5 / 48(10)$ \\
\hline
\end{tabular}

Values are mean \pm standard deviation or number of patients (percentage of total number of patients) or median (interquartile range).

SOFA: Sequential organ failure assessment; ICU: Intensive Care Unit; BMI: Body mass index
Relation of intra-abdominal pressure with acute kidney injury

Early AKI occurred in 54\% (26/48) patients. The IAP was statistically similar between patients with and without early AKI on day $1(9.1 \pm 2.1$ vs. $8.6 \pm 3.8 \mathrm{mmHg} ; P=0.542)$ as well as day $2(8.1 \pm 2.2$ vs. $8.2 \pm 2.8 ; P=0.907)$ of ICU admission.

The incidence of IAH during ICU stay was also statistically similar in patients with and without early AKI ( $5 / 26=19 \%$ vs. $5 / 22=23 \%$, respectively) $(P=0.766)$.

ROC curves were constructed for correlation of early AKI with the IAP on day 1 as well as day 2 of ICU stay; the area under curve (AUC) on day 1 was 0.499 (95\% confidence interval [CI]: $0.325-0.673)(P=0.992)$ and on day 2 was 0.474 (95\% CI: $0.280-0.668)(P=0.788)$.

\section{Relation of urinary neutrophil gelatinase-associated} lipocalin with acute kidney injury

Urinary NGAL concentration was significantly greater in patients with early AKI (86 [46-98] ng/ml) as compared to those without AKI $(38[5-66] \mathrm{ng} / \mathrm{ml})(P=0.006)$.

A ROC curve was constructed for correlation of urinary NGAL with the occurrence of early AKI [Figure 1]. The association was significant with an AUC of 0.734 (95\% CI: $0.583-0.884)(P=0.006)$. The optimal cutoff NGAL value at $53.7 \mathrm{ng} / \mathrm{ml}$ had a sensitivity and specificity of $73 \%$ each.

Observations related to intra-abdominal pressure and epidemiology of intra-abdominal hypertension

Incidence of IAH was $10 / 48(21 \%)$. In patients with IAH, the IAP ranged from 12 to $14 \mathrm{mmHg}$ depicting Grade I of severity in all $(12-15 \mathrm{mmHg})$.

Of the various major and minor risk factors evaluated for association with the development of IAH, the incidence of abdominal distension, intraperitoneal infection, and massive fluid resuscitation was significantly greater in patients with

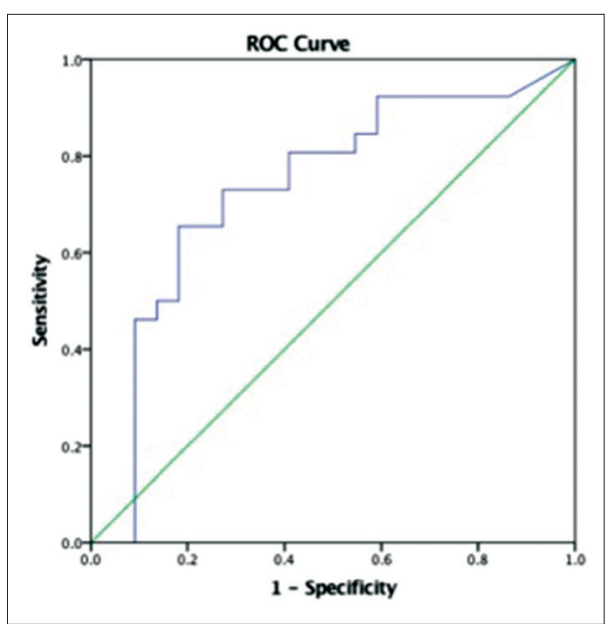

Figure 1: Diagnostic performance of urinary neutrophil gelatinase-associated lipocalin for predicting early acute kidney injury based on the calculation of the area under the receiver operating characteristic curve 
IAH as compared to those without IAH $(P<0.05)$ [Table 2]. All other risk factors were similarly distributed between patients with and without IAH $(P>0.05)$ [Table 2]. The only patient who had not delivered before ICU admission developed IAH.

The SOFA score on admission was clinically greater but statistically similar between patients with and without IAH (9 [4.7-11] and $6[4-8] ; P=0.128)$.

ICU mortality was clinically higher, but statistically similar between patients with and without IAH $(2 / 10=20 \%$ vs. $3 / 38=8 \%$, respectively) $(P=0.265)$.

Median value of the highest IAP recorded for each individual patient over the entire duration of ICU stay was 9 [7-11.5] $\mathrm{mmHg}$.

The IAP was measured daily over first 7 days of ICU stay. The trend of median values of daily IAP was calculated for the first 5 days only, since on the $6^{\text {th }}$ and $7^{\text {th }}$ day, only 3 and 2 patients, respectively, remained in the ICU. The median values varied from 6.5 to $9 \mathrm{mmHg}$, showing a progressive decrease over time [Table 3].

\section{Observations related to early acute kidney injury}

Of the 26 patients who developed early AKI, 12 (46\%) patients developed a mild form, that is, Stage 1 of severity, while $8(31 \%)$ and $6(23 \%)$ had Stage 2 and 3, respectively.

Mortality was clinically higher but statistically similar for patients with and without AKI ( $4 / 26=15.4 \%$ vs. $1 / 22=4.5 \%$, respectively) $(P=0.221)$. Mortality in those with moderate or severe form of AKI, that is, Stage 2 or 3 was clinically as well as significantly higher than those without it $(4 / 14=29 \%$ vs. $1 / 34=3 \%$, respectively) $(P=0.008)$.

\section{Discussion}

The present cohort study found a lack of any association between IAP and early AKI in critically ill obstetric patients. This was despite AKI and IAH being common in these patients (54\% and $21 \%$, respectively). Urinary NGAL measured at time of ICU admission proved to be reliable for detecting early AKI. A cutoff value of $53.7 \mathrm{ng} / \mathrm{ml}$ is associated with fairly good sensitivity and specificity ( $73 \%$ each).

Increase in IAP is known to affect the renal function adversely. ${ }^{[4,10-12]}$ Demarchi et al. recently noted IAP values of $>8 \mathrm{mmHg}$ to predict the development of AKI. ${ }^{[13]}$ Contrastingly, we noted a lack of effect of IAP on early AKI when evaluating the relationship in critically ill obstetric patients. In obstetric patients, it is not established whether the raised IAP values are truly a pathologic elevation or merely a physiological change secondary to the mechanical effects of a growing fetus. ${ }^{[6]}$ There is very little previous data analyzing the effect of IAP on organ function and herein a lack of effect on SOFA score was observed. ${ }^{[6]}$ Although the current guidelines recommend intravesical bladder catheter to be used for measuring IAP in all patients, ${ }^{[1]}$ the validity of the technique has been questioned for obstetric subset. ${ }^{[7]}$ The

\begin{tabular}{|c|c|c|c|}
\hline Characteristic & $\begin{array}{c}\text { With IAH } \\
(n=10), n(\%)\end{array}$ & $\begin{array}{l}\text { Without IAH } \\
(n=38), n(\%)\end{array}$ & $P$ \\
\hline Abdominal distension & $4(40)$ & $2(5)$ & 0.003 \\
\hline Intraperitoneal infection & $1(10)$ & 0 & 0.049 \\
\hline Massive fluid resuscitation & $1(10)$ & 0 & 0.049 \\
\hline Liver dysfunction & 0 & $1(3)$ & 0.604 \\
\hline Polytransfusion & 0 & $2(5)$ & 0.459 \\
\hline Sepsis & $1(10)$ & $6(16)$ & 0.644 \\
\hline Coagulopathy & $1(10)$ & $5(13)$ & 0.788 \\
\hline Acidosis & $3(30)$ & $2(5)$ & 0.924 \\
\hline Abdominal surgery & $9(90)$ & $31(82)$ & 0.525 \\
\hline Major trauma & 0 & 0 & - \\
\hline Ileus & 0 & 0 & - \\
\hline Mechanical ventilation & $10(100)$ & $32(84)$ & 0.179 \\
\hline PEEP $>10 \mathrm{cmH}_{2} \mathrm{O}$ & 0 & $2(5)$ & 0.459 \\
\hline $\mathrm{BMI}>30 \mathrm{~kg} / \mathrm{m}^{2}$ & 0 & $1(3)$ & 0.604 \\
\hline Hypothermia & 0 & 0 & - \\
\hline $\begin{array}{l}\text { Pregnant at time of ICU } \\
\text { admission }\end{array}$ & $1(10)$ & 0 & 0.049 \\
\hline
\end{tabular}

Table 3: Trend of daily intra-abdominal pressure

\begin{tabular}{lc}
\hline Characteristic & Value $(\mathbf{m m H g})$ \\
\hline Day $1(48)$ & $9(7-11.5)$ \\
Day 2 (37) & $8(6.5-10.5)$ \\
Day 3 (21) & $8(6.5-9.7)$ \\
Day 4 (13) & $7.5(5.7-8.7)$ \\
Day 5 (6) & $6.5(5.6-9.3)$ \\
\hline Number of patients remaining in the Intensive Care Unit indicated in \\
parentheses ( ); Values are median (interquartile range)
\end{tabular}

weight of the gravid uterus on the bladder is hypothesized to spuriously augment the IAP. Although all, except one of our patients were in postpartum period, the temporal changes in uterine size, abdominal wall compliance, and proximity of the bladder to a progressively decreasing uterus could all be affecting the IAP. All of these factors could have contributed to the lack of effect on IAP on renal function.

We also evaluated the epidemiology of IAH in critically ill obstetric patients. IAH was common (21\%) and occurrence of abdominal distension, intraperitoneal infection, massive fluid resuscitation ( $>3.5 \mathrm{l} / \mathrm{day})$, and presence of the fetus in utero were significantly higher in those with IAH as compared to without it. It was associated with worse organ functions depicted by greater SOFA score and increased ICU mortality, although statistically similar.

We observed urinary NGAL done at the time of ICU admission to be significantly higher in patients who developed early AKI and have a fair discriminatory ability to predict early AKI. The area under ROC curve was comparable to previous studies evaluating the use of urinary NGAL for prediction of AKI in 
critically ill patients. ${ }^{[14,15]}$ Although the ability of urinary NGAL to predict AKI in critically ill patients is well documented, we could not locate any data for critically ill obstetric patients. There are studies evaluating urinary NGAL during pregnancy as a marker of severity of preeclampsia or to predict AKI in preeclamptics in particular. ${ }^{[16,17]}$ Pregnancy is associated with a decrease in normal serum creatinine values due to the physiologic changes. In such a scenario, urinary NGAL could thus serve as a useful biomarker for renal dysfunction in these patients.

The incidence of IAH (21\%) noted by us is higher than previous findings published very recently by our group $(6 \%) .{ }^{[6]} \mathrm{We}$ cannot attribute this to a single concrete reason, however, a higher volume was instilled in the bladder in the current study $(25 \mathrm{ml}$ vs. $20 \mathrm{ml})$ and the sample size of previous study was larger. The higher prevalence of IAH noted by us in this study is closer to figures quoted for mixed populations of critically ill patients in the ICU. ${ }^{[3,10]}$ The occurrence of IAH was significantly associated with abdominal distension, intraperitoneal infection, massive fluid resuscitation, and continued pregnancy at time of ICU admission. These are previously well-documented risk factors associated with the development of IAH. ${ }^{[1]}$ The SOFA score was worse and mortality higher (20\% vs. $8 \%$ ) in patients with IAH. It probably failed to achieve statistical significance since the cohort was not powered to study such adverse effect of IAH that is previously established.

The incidence of early AKI in critically ill obstetric patients was noted to be $54 \%$ in our study. Earlier studies have reported a much lower incidence of AKI in critically ill obstetric patients $(6 \%$ and $33 \%))^{[18,19]}$ The probable reason for higher incidence of AKI evidenced by us would be the use of KDIGO criteria as compared to RIFLE criteria used in both previous studies. The KDIGO criteria consider Stage 1 AKI as even a small increase in serum creatinine of $\geq 0.3 \mathrm{mg} /$ $\mathrm{dl}$ in a $48 \mathrm{~h}$ period, which is not included in the initial stage of RIFLE criteria. This is corroborated by $46 \%(12 / 26)$ of our patients with AKI to be in Stage 1 of the disease. If we were to consider only Stage 2 and 3 patients, the resultant incidence of $10 / 26(20 \%)$ would be similar to that reported earlier. ${ }^{[19]}$

We observed significantly higher ICU mortality of $29 \%$ in patients with moderate-to-severe AKI as compared to $3 \%$ in those without AKI. AKI contributing to increased mortality is previously well established. ${ }^{[8]}$ Mortality rate among patients with AKI noted by us is similar to that reported by Bentata et al. in critically ill obstetric patients with AKI. ${ }^{[19]}$

A limitation of the present cohort may be its evaluation of AKI only over the early period and not over the entire duration of ICU stay. Furthermore, besides IAP, other proven risk factors leading to AKI may have contributed to its development. However, the present study did not aim to evaluate risk factors for AKI per se.

\section{Conclusions}

Although early AKI is common in critically ill obstetric patients, it is not related to IAP. Urinary NGAL is a good aid to diagnose early AKI in critically ill obstetric patients. Such indicators of AKI that rise early could help in early diagnosis and intervention thus improving patient outcome.

\section{Financial support and sponsorship}

Nil.

\section{Conflicts of interest}

There are no conflicts of interest.

\section{RefEREnCES}

1. Kirkpatrick AW, Roberts DJ, De Waele J, Jaeschke R, Malbrain ML, De Keulenaer B, et al. Intra-abdominal hypertension and the abdominal compartment syndrome: Updated consensus definitions and clinical practice guidelines from the world society of the abdominal compartment syndrome. Intensive Care Med 2013;39:1190-206.

2. Streat SJ. Abdominal surgical catastrophes. In: Bersten AD, Soni N, editors. Oh's Intensive Care Manual. $6^{\text {th }}$ ed. Philadelphia: Butterworth Heinmann Elsevier; 2000. p. 499-508.

3. Malbrain ML, Chiumello D, Cesana BM, Reintam Blaser A, Starkopf J, Sugrue M, et al. A systematic review and individual patient data meta-analysis on intra-abdominal hypertension in critically ill patients: The wake-up project. World initiative on abdominal hypertension epidemiology, a unifying project (WAKE-up!). Minerva Anestesiol 2014;80:293-306.

4. Armaly Z, Abassi Z. Deleterious effects of increased intra-abdominal pressure on kidney function. Adv Nephrol 2014;2014:731657. doi: $10.1155 / 2014 / 731657$.

5. Singer E, Markó L, Paragas N, Barasch J, Dragun D, Müller DN, et al. Neutrophil gelatinase-associated lipocalin: Pathophysiology and clinical applications. Acta Physiol (Oxf) 2013;207:663-72.

6. Tyagi A, Singh S, Kumar M, Sethi AK. Intra-abdominal pressure and intra-abdominal hypertension in critically ill obstetric patients: A prospective cohort study. Int J Obstet Anesth 2017;32:33-40.

7. Chun R, Kirkpatrick AW. Intra-abdominal pressure, intra-abdominal hypertension, and pregnancy: A review. Ann Intensive Care 2012;2 Supp1 1:S5.

8. Kidney Disease: Improving Global Outcome. Available from: http://www.kdigo.org. [Last accessed on 2015 Sep 15].

9. Bellomo R, Ronco C, Kellum JA, Mehta RL, Palevsky P, Acute Dialysis Quality Initiative workgroup. et al. Acute renal failure - Definition, outcome measures, animal models, fluid therapy and information technology needs: The second international consensus conference of the acute dialysis quality initiative (ADQI) group. Crit Care 2004;8:R204-12.

10. Malbrain ML, Chiumello D, Pelosi P, Bihari D, Innes R, Ranieri VM, et al. Incidence and prognosis of intra-abdominal hypertension in a mixed population of critically ill patients: a multiple-center epidemiological study. Crit Care Med 2005;33:315-22.

11. Biancofiore G, Bindi ML, Romanelli AM, Bisà M, Boldrini A, Consani $\mathrm{G}$, et al. Postoperative intra-abdominal pressure and renal function after liver transplantation. Arch Surg 2003;138:703-6.

12. Cullen DJ, Coyle JP, Teplick R, Long MC. Cardiovascular, pulmonary, and renal effects of massively increased intra-abdominal pressure in critically ill patients. Crit Care Med 1989;17:118-21.

13. Demarchi AC, de Almeida CT, Ponce D, e Castro MC, Danaga AR, Yamaguti FA, et al. Intra-abdominal pressure as a predictor of acute kidney injury in postoperative abdominal surgery. Ren Fail 2014;36:557-61.

14. Kamis F, Yegenaga I, Musul M, Baydemir C, Bek S, Kalender B, et al. Neutrophil gelatinase-associated lipocalin levels during the first 48 hours of intensive care may indicate upcoming acute kidney injury. J Crit Care 2016;34:89-94. 
15. Matsa R, Ashley E, Sharma V, Walden AP, Keating L. Plasma and urine neutrophil gelatinase-associated lipocalin in the diagnosis of new onset acute kidney injury in critically ill patients. Crit Care 2014;18:R137.

16. Simonazzi G, Capelli I, Curti A, Comai G, Rizzo N, La Manna G, et al. Serum and urinary neutrophil gelatinase-associated lipocalin monitoring in normal pregnancy versus pregnancies complicated by pre-eclampsia. In Vivo 2015;29:117-21.

17. Moyake N, Buchmann E, Crowther NJ. Neutrophil gelatinase-associated lipocalin as a diagnostic marker of acute kidney injury in pre-eclampsia J Obstet Gynaecol Res 2016;42:1483-8.

18. Kamal EM, Behery MM, Sayed GA, Abdulatif HK. RIFLE classification and mortality in obstetric patients admitted to the Intensive Care Unit with acute kidney injury: A 3-year prospective study. Reprod Sci 2014;21:1281-7.

19. Bentata Y, Housni B, Mimouni A, Azzouzi A, Abouqal R. Acute kidney injury related to pregnancy in developing countries: Etiology and risk factors in an Intensive Care Unit. J Nephrol 2012;25:764-75. 